\title{
Dynamische Demokratie als Herausforderung und Ziel: Prolegomena zur Analyse von Demokratiereformen
}

\author{
von Ludger Helms
}

\begin{abstract}
Die liberale Demokratie ist eine außerordentlich dynamische Staats- und Herrschaftsform, die sich wandelt, auch wenn man sie nicht reformiert. Reformen stellen einen Weg dar, dem Wandel der Demokratie eine bestimmte Richtung zu geben. Während Reformen an der Demokratie politisch in aller Munde sind, ist die politikwissenschaftliche Beschäftigung mit Demokratiereformen vergleichsweise wenig entwickelt. Der Komplexität der liberalen Demokratie vermögen nur Ansätze gerecht zu werden, die neben den institutionellen Optionen die jeweils vorherrschenden politischen, gesellschaftlichen und kulturellen Bedingungen von Demokratie und die jeweiligen Kompatibilitätspotentiale zwischen institutionellen Lösungen und gesellschaftichen Erwartungen berücksichtigen. Die Gesellschaft ist in ihrer Bedeutung für die Demokratiereform jedoch nicht auf den Status einer erklärenden Variable oder kontextuellen Randbedingung beschränkt; sie ist vielmehr selbst ein mögliches Objekt demokratiepolitischer Reformen.
\end{abstract}

Liberal democracy represents an immensely dynamic type of political regime, which tends to evolve and change even in the absence of political reform. Reforms may be considered mechanisms designed to give change a particular direction. Whereas democratic reforms are widely discussed in many contemporary democracies, social scientific analyses thereof have remained rather thin on the ground. When analysing democratic reforms, the complexity of liberal democracy requires taking into account both the institutional and social/cultural conditions of democratic governance as well as the compatibility between institutional solutions and social expectations. That said, society is far from being a mere contextual component of reform policies or an explanatory variable for democratic reforms; rather, it marks a possible target of political reforms in its own right.

\section{Einleitung}

Politische Reformen sind aus der Politik und der politischen Rhetorik demokratischer Gemeinwesen nicht wegzudenken. Genau genommen war das immer so. Gleichwohl vermitteln viele der jüngeren Beiträge vor allem aus dem politischen Journalismus den Eindruck, dass die jüngere Vergangenheit geradezu so etwas 
wie ein postmodernes „Reformzeitalter“, seine Protagonisten Mitglieder einer „Generation Reform“" seien. Wo von politischen Reformen gesprochen wird, sind zumeist Reformen auf unterschiedlichen Politikfeldern gemeint, ganz besonders im Bereich des Sozialstaates, der in fast allen Ländern Europas seit einigen Jahren nachhaltig restriktive Modifikationen erfährt. Mit Blick auf politische Reformen dieser Art hat die internationale Politikwissenschaft wichtige Differenzierungen angeboten, die sich vor allem an der Reichweite der Veränderung orientieren, die mit Reformen angestrebt bzw. erreicht werden. ${ }^{2}$ Auch ausgreifende ländervergleichende Betrachtungen zur politischen Reformfähigkeit der Demokratie sind mehrheitlich auf unterschiedliche Politikfelder bezogen. ${ }^{3}$

Im Zentrum dieses Beitrags stehen nicht solche politischen Reformen in der Demokratie, sondern vielmehr Reformen an der Demokratie, wobei die Schnittmenge zwischen beiden freilich beträchtlich ist. Die Grundlage der Beschäftigung mit Reformen an der Demokratie bildet die Überzeugung von deren Bewahrens- und Reformwürdigkeit. Im Lichte der jüngeren Debatte über die Vorzüge und Kosten der Demokratie betrachtet, scheint diese Überzeugung auch in vielen konsolidierten liberalen Demokratien an Selbstverständlichkeit verloren zu haben. Sorgfältige Rekonstruktionen der Argumente der Gegner der Demokratie $^{4}$ und empirische Leistungsvergleiche zwischen demokratischen und nichtdemokratischen Regimen ${ }^{5}$ lassen jedoch keinen Zweifel daran, dass die liberale Demokratie, trotz aller erkennbaren Schwächen, mit großem Abstand die Regierungsform mit dem größten humanen Potential ist.

Jene, die diese Überzeugung teilen, haben sich dem Gegenstand aus sehr unterschiedlichen Perspektiven genähert. Zum 60. Bestehen des Grundgesetzes mangelt es in Deutschland nicht an Beiträgen, die die Leistungen verfassungsrechtlicher Institutionen für die Stabilität und Wohlfahrt der Demokratie betonen und teils aus stärker politischer, teils aus genuin wissenschaftlicher Perspektive - die spezifischen Errungenschaften der deutschen Nachkriegsverfassung wie die

1 Nolte, P.: Generation Reform: Jenseits der blockierten Republik, München, 2004.

2 Hall, P.A.: Policy Paradigms, Social Learning, and the State, in: Comparative Politics, 25/3 (1993), 239-255

3 Vorländer, H. (Hg.): Politische Reformen in der Demokratie, Baden-Baden 2005; Delhees, S. u. a.: Wohlfahrtsstaatliche Reformkommunikation: Westeuropäische Parteien auf Mehrheitssuche, BadenBaden, 2008; Wagschal, U. (Hg.): Deutschland zwischen Reformstau und Veränderung. Ein Vergleich der Politik- und Handlungsfelder, Baden-Baden, 2009 (i. E.).

4 Gilley, B.: Is Democracy Possible?, in: Journal of Democracy, 20/1 (2009), 113-127.

5 Schmidt, M. G.: Wie leistungsfähig ist die Demokratie?, in: Mohr, A./Nohlen, D. (Hg.): Politikwissenschaft in Heidelberg, Heidelberg, 2008, 205-214. 
offen gebliebenen und neu hinzugekommenen Fragen beleuchten. ${ }^{6}$ Dabei verbindet die meisten Autoren die Einsicht, dass „Verfassungen [...] etwas Gegebenes, zugleich aber auch etwas zur Weiterentwicklung Aufgegebenes“" ${ }^{\text {“7 }}$ sind, wobei es sich bei vielen Aufgaben der Zukunft selbst in den Augen rechtwissenschaftlicher Betrachter weniger um Herausforderungen verfassungsrechtlicher als vielmehr politischer Natur handelt. ${ }^{8}$

Bei einer Reihe jüngerer internationaler Beiträge zum Themenkreis Demokratiereform handelt es sich um groß angelegte, phantasievolle Entwürfe für eine Vervollkommnung der liberalen Demokratie. ${ }^{9}$ Ihnen ist nur bedingt das Zeugnis hinreichender empirischer Bodenhaftung auszustellen; jedenfalls werden Fragen und Probleme reformpolitischer „Machbarkeit“ häufig nur am Rande erörtert. Gerade dieser operative Aspekt wird von den Vertretern eines anderen Paradigmas besonders großgeschrieben: Gleichsam als „Startschuss“ der jüngeren deutschsprachigen Debatte auf diesem Feld kann ein programmatischer Beitrag aus der Feder Joachim Jens Hesses gelten. ${ }^{10}$ Wie in zahlreichen späteren Beiträgen des Autors wird darin gefordert, sich im Rahmen einer realitätsorientierten Analyse auf „die Komplexität der ,arbeitenden“ Institutionen“ einzulassen und dabei, unter Berücksichtigung normativer und funktionaler Aspekte, nicht zuletzt die praktische Relevanz des eigenen Tuns im Blick zu behalten. ${ }^{11}$ In demselben

6 Aus der wissenschaftlichen Literatur seien (statt vieler) genannt: Oppermann, T.: Deutschland in guter Verfassung? 60 Jahre Grundgesetz, in: Juristenzeitung, 64/10 (2009), 481-532; Sachs, M.: Das Grundgesetz in seinem sechsten Jahrzehnt, in: Neue Juristische Wochenschrift, 62/21 (2009), 1441-1536; Vorländer, H.: Die Deutschen und ihre Verfassung, in: Aus Politik und Zeitgeschichte, B 18-19/2009, 818; Dreier, H.: Das Grundgesetz - eine Verfassung auf Abruf?, in: Aus Politik und Zeitgeschichte, B 18-19/2009, 19-26.

7 So Voßkuhle, A.: NJW-Editorial: 60 erfolgreiche Jahre Grundgesetz - kein Grund, sich auszuruhen!, in: Neue Juristische Wochenschrift, 62/21 (2009), III.

8 Vgl. dezidiert Oppermann, T., a. a. O., 491.

9 Vgl. etwa Schmitter, P.C.: Wie könnte eine ,postliberale Demokratie“ aussehen? Skizzenhafte Vermutungen und Vorschläge, in: Offe, C. (Hg.): Demokratisierung der Demokratie. Diagnosen und Reformvorschläge, Frankfurt a. M./New York, 2003, 152-165; Bassey, S. E.: No-Party Parliamentary Democracy: The Ideal Political System for the New Age, Bloomington, IN, 2005; Goodin, R.: Innovating Democracy: Democratic Theory and Practice After the Deliberative Turn, Oxford, 2008; Smith, G.: Democratic Innovations: Designing Institutions for Citizen Participation, Cambridge, 2009.

10 Hesse, J.J.: Aufgaben einer Staatslehre heute, in: Jahrbuch zur Staats- und Verwaltungswissenschaft, 1 (1987), 55-87.

11 Ebd., 83; stets geht es darum, ,funktionsorientierte Analysen mit demokratietheoretischen Erwägungen und operativer Kompetenz zu verbinden“" und dabei zugleich die traditionellen Grenzen zwischen den beteiligten akademischen Teildisziplinen zum Wohle der Erkenntnismaximierung zu überwinden; vgl. Hesse, J.J.: Editorial, in: ZSE, 4/1 (2006), 3-8, hier 6. - Eine Vorstellung davon, wie weit dieser Weg noch sein mag, vermittelt eine vielbeachtete kleine Monographie von Möllers, C.: Der vermisste Leviathan, Frankfurt a. M., 2008. Das erklärte Ziel der Untersuchung besteht darin, im Zuge der Auseinander- 
Band findet sich ein wichtiger Beitrag Fritz W. Scharpfs, in dem (bereits einige Jahre vor Beginn der internationalen Konjunktur des ,,comparative institutional engineering") die Möglichkeiten und Grenzen institutioneller Reformen beleuchtet werden. ${ }^{12}$ Reformen erscheinen dabei als das (mögliche) Ergebnis eines „reformorientierte(n), aktive(n) Management(s) von Konfigurationsprozessen“. ${ }^{13}$ Ein solches Verständnis von Reformpolitik mag für einen akteurstheoretisch angeleiteten und nach Praxisrelevanz strebendem Zugang als gleichsam ,natürliche“, wenn auch bemerkenswert komplexe Sicht der Dinge erscheinen. Weitaus revolutionärer ist die These Scharpfs, ,dass der Prozess der institutionellen Reform nach der politischen Durchsetzung, Verabschiedung und Implementation überhaupt erst beginnt“14 . Damit wird das Tor zur systematischen Einbeziehung gesellschaftlicher Aspekte politischer Reformen weit aufgestoßen, ohne dass es den Anschein hätte, als wenn seither viele durch dieses geschritten wären. ${ }^{15}$

Die in diesem Beitrag angestellten Betrachtungen stehen in der Tradition von Arbeiten, in denen es auf breiter Grundlage um die Rekonstruktion, Erklärung und Bewertung der Prozesse und Ergebnisse von Staats- und Demokratiereformen geht. ${ }^{16}$ Dabei wird die zentrale Bedeutung operativer Aspekte einer Be-

setzung mit unterschiedlichen Perspektiven auf den Staat ,namentlich nach Anschlussdiskussionen mit den Politikwissenschaften zu suchen“; ebd.: 11. Gleichsam als Königsweg der Erkenntnis wird dabei der internationale Vergleich herausgestellt. Dass Möllers die grundlegende Einsicht, komparativ relevantes Wissen entspringe nicht ausschließlich den Rechtswissenschaften, sondern auch anderen Disziplinen wie etwa der Vergleichenden Regierungslehre, einem Dritten verdankt (vgl. die Danksagung in FN 2 auf S. 116), selbst aber offenbar nicht einen einzigen weiteren Satz über diesen Gegenstand zu sagen weiß, grenzt an das unfreiwillig Komische. Einen positiveren Eindruck von der Bereitschaft und Fähigkeit zur Öffnung der Rechtswissenschaften vermitteln einige Beiträge in Engel, C./Schön, W. (Hg.): Das Proprium der Rechtswissenschaft, Tübingen, 2007 und Jestaedt, M./Lepsius, O. (Hg.): Rechtwissenschaftstheorie, Tübingen, 2008.

12 Scharpf, F. W.: Grenzen institutioneller Reform, in: Jahrbuch zur Staats- und Verwaltungswissenschaft, 1 (1987), 111-151.

13 Ebd., 148.

14 Ebd., 144.

15 Die ,governance“-Literatur kommt diesem Anspruch (zumindest auf den ersten Blick) am nächsten. Allerdings scheinen bei ihr andererseits sowohl die Einsicht in das konkrete Regierungs- und Verwaltungshandeln als auch das Interesse an demokratietheoretischen Fragen verbreitet eher gering zu sein. Hinzu kommen gravierende terminologisch-konzeptuelle Probleme, die einem systematischen Erkenntnisfortschritt im Wege stehen und die politisch-gesellschaftliche Relevanz vieler Arbeiten dieser Richtung herabsetzen. Vgl. Offe, C.: Governance - „Empty signifier“ oder sozialwissenschaftliches Forschungsprogramm?, in: Schuppert, G.F./Zürn, M. (Hg.): Governance in einer sich wandelnden Welt (PVS-Sonderheft 41/2008), Wiesbaden, 2008, 61-76.

16 Vgl. etwa Cain, B./Dalton, R.J./Scarrow, S. (Hg.): Democracy Transformed? Expanding Political Opportunities in Advanced Democracies, Oxford, 2003; Seidle, F.L./Docherty, D. C. (Hg.): Reforming Parliamentary Democracy, Montreal/Kingston, 2003; Offe, C. (Hg.): Demokratisierung der Demokratie, 
schäftigung mit Reformen demokratischer Gemeinwesen keineswegs geleugnet. Allein der notwendigerweise beschränkte Umfang der Untersuchung bedingt die bevorzugte Behandlung ausgewählter Grundlagenaspekte des Themas, die ihrerseits stärker praxeologisch ausgerichtete Analysen befruchten mag. ${ }^{17}$

\section{Zum Konzept der liberalen Demokratie}

Am Beginn muss eine kurze Verständigung über die „liberale Demokratie“ stehen. Diese wird hier nicht als policy-bezogener Regimetyp begriffen, der so etwas wie ein neo-liberales Gegenmodell zur „sozialen Demokratie“ darstellen würde ${ }^{18}$, sondern als Grundtypus einer Herrschaftsform, die prinzipiell offen ist für gravierend unterschiedliche Ausgestaltungen auf der Ebene der Staatstätigkeit.

Diese basale Differenzierung führt freilich keineswegs ohne weiteres zu einer konsensfähigen Grundauffassung über die Merkmale der liberalen Demokratie als Herrschaftsform. Die liberale Demokratie bleibt nicht nur politisch, sondern auch konzeptuell umstritten, begründungs- und erklärungsbedürftig. Als grundlegendes Strukturmerkmal liberaler Demokratie als Regimetyp kann die Verbindung von liberalen mit demokratischen Elementen gelten. Nicht zufällig werden demokratische Verfassungsstaaten auch als liberal-demokratische Systeme bezeichnet. ${ }^{19}$ Die grundlegenden Referenzwerte der liberalen Demokratie - Freiheit und Gleichheit - betreffen, „durchwirken“ jeweils beide dieser konstitutiven Komponenten bzw. Dimensionen der liberalen Demokratie: Mit Blick auf das Prinzip der Freiheit heißt das etwa: Freiheit vor dem Staat (durch Menschenrechte und subjektive Rechte) und Gewaltenteilung als wichtige Aspekte der liberalen, rechtsstaatlichen Dimension - freie Wahlen, Versammlungsfreiheit und

a.a.O.; Beck, R.A./Schaller, C.: Zur Qualität der österreichischen und britischen Demokratie: empirische Befunde und Anregungen für Demokratiereform, Wien u. a., 2003.

17 Bei diesem Beitrag handelt es sich gleichsam um eine Vorstudie zu einem größeren Projekt des Verfassers zum Thema „Demokratiereform“, das im kommenden Jahr im Verlag C.H. Beck, München erscheinen wird. Vgl. zur Diskussion über das Spannungsverhältnis von Staatstheorie und Staatspraxis auch das Themenheft 3/2009 der ZSE.

18 Streeck, W.: Einleitung: Internationale Wirtschaft, nationale Demokratie?, in: Streeck, W. (Hg.): Internationale Wirtschaft, nationale Demokratie: Herausforderungen für die Demokratietheorie, Frankfurt a. M./New York, 1998, 11-58, hier 13, 18.

19 Shell, K. L.: Liberal-demokratische Systeme: eine politisch-soziologische Analyse, Stuttgart u.a., 1981. Aus der Verbindung von liberalen und demokratischen Elementen entspringt in funktionaler Hinsicht die doppelte Eigenschaft von liberal-demokratischen Regimen als ,,limited government “ und ,,limited democracy“; vgl. Helms, L.: Die Institutionalisierung der liberalen Demokratie, Frankfurt a. M./New York, 2007, $18 \mathrm{f}$. 
weiteres als zentrale Aspekte der demokratischen, im Kern partizipationsbezogenen Komponenten von Demokratie. Entsprechend gilt mit Blick auf das Gleichheitsprinzip: Gleichheit vor dem Gesetz und des Zugangs zur Justiz als Aspekte der liberalen Dimension, Gleichheit der Partizipationsrechte und Zählwertgleichheit von Stimmen als Ausdruck des Gleichheitsprinzips auf der demokratischen Ebene. $^{20}$

Als konsolidierte liberale Demokratien werden solche Gemeinwesen bezeichnet, in denen sich die Demokratie über einen längeren Zeitraum hinweg als stabil erwiesen und auch gesellschaftliche Wurzeln geschlagen hat. ${ }^{21}$ Selbst für die konsolidierten liberalen Demokratien gilt jedoch - und dieser Punkt ist für die weiteren Betrachtungen von besonderer Relevanz -, dass es sich bei ihnen um hochgradig dynamische Gebilde handelt. In der Tat könnte man das Dynamische geradezu als ein „Epitheton ornans der Demokratie“22 bezeichnen. Potentiell kann sich alles wandeln, was die Demokratie ausmacht: die Werte und Verhaltensweisen auf der Ebene der Gesellschaft und der Eliten ebenso wie die politisch-gesellschaftlichen Kräfteverhältnisse bis hin zu den grundlegenden Institutionen der Demokratie selbst. Die charakteristische Dynamik demokratischer Regierungssysteme speist sich jedoch nicht allein aus unterschiedlichen internen Quellen der Veränderung, sondern ist auch dem Umstand geschuldet, dass Demokratien stets in ein spezifisches internationales bzw. transnationales Umfeld eingebettet sind, das ebenfalls hochgradig dynamisch ist und von dem wiederum ganz unterschiedliche Impulse des Wandels ausgehen können. Dazu gehören stärker gesellschaftliche Aspekte wie die internationale Migration ebenso wie ökonomische und stärker politisch-regulative Aspekte wie sie die große Mehr-

20 Als nicht zielführend betrachtet werden können die Konzeptualisierungen bei Bühlmann, M. u. a.: Wie lässt sich Demokratie am besten messen? Zum Forumsbeitrag von Thomas Müller und Susanne Pickel, in: Politische Vierteljahresschrift, 49/1 (2008), 114-122, hier 118. Dort werden als zentrale Komponenten des Prinzips „Freiheit“ die Gleichheit vor dem Gesetz und die Gleichheit des Zugangs zur Justiz genannt, als zentrale Komponenten des Prinzips „Gleichheit“ hingegen unter anderem die Versammlungsfreiheit und die Meinungsfreiheit sowie eine aktive Öffentlichkeit.

21 Diamond, L. u. a. (Hg.): Consolidating the Third Wave Democracies: Themes and Perspectives, Baltimore/London, 1997.

22 Neisser, H.: Demokratiereform in Österreich: Potentiale und Instrumente, in: Pelinka, A./Plasser, F./Meixner, W. (Hg.): Die Zukunft der österreichischen Demokratie. Trends, Prognosen und Szenarien, Wien, 2000, 423-438, hier 421; mit einem starken politischen Impuls entwickelt diesen Aspekt Pelinka, A.: Dynamische Demokratie. Zur konkreten Utopie gesellschaftlicher Gleichheit, Stuttgart u. a., 1974. 
zahl demokratischer Regime in Europa vor allem in Gestalt der europäischen Integration betreffen. ${ }^{23}$

\section{Dimensionen politischer Reformen}

Es geht hier jedoch nicht um jede mögliche Veränderung der Parameter liberaler Demokratien, sondern spezieller um politische Reformen. Dabei handelt es sich freilich um einen schillernden Begriff. Klaus Dieter Wolf versteht darunter, „durch politische Entscheidungen bewusst herbeigeführte Veränderungen von

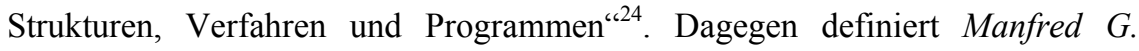
Schmidt Reformen als „die Planung, den Prozess und das Ergebnis von Bestrebungen“, „mit legalen Mitteln bestehende Strukturen neu zu ordnen“25. Diese zweite Definition ist ein wenig breiter und spezifischer zugleich: Sie ist breiter, insofern sie auch die üblicherweise hoch komplexen politischen Willensbildungs- und Planungsprozesse ausdrücklich mit abdeckt; sie ist spezifischer, insofern sie Reformen ausdrücklich als solche Bestrebungen der Neuordnung charakterisiert, die sich legaler Mittel bedienen, womit der Begriff „Reform“ von jenem der „Revolution“ abgesetzt ist. ${ }^{26}$ Daneben gibt es freilich „politischere“ Reformverständnisse, die als politische Reform nur solche Strukturveränderungen gelten lassen, die, auf die eine oder andere Weise, eine Umverteilung von Macht einschließen. ${ }^{27}$ Die Frage, ob bzw. in welchem Maße dieses Kriterium gewährleistet ist, lässt sich nicht immer leicht beantworten: Evident ist der Aspekt der Machtverschiebung vor allem im Kontext von Reformen an Wahlsystemen, die treffend als ,redistributive Institutionen ${ }^{، 28}$ beschrieben wurden. ${ }^{29}$ In vielen anderen Bereichen der Demokratiereform ist der Machtaspekt weniger direkt greifbar, jedoch selten wirklich abwesend.

23 Helms, L.: The Liberal-Democratic Foundations of the European Nation-State and the Challenges of Internationalization, in: International Politics, 46/1 (2009), 48-64.

24 Wolf, K-D.: Einführungsvortrag: Staat und Gesellschaft - fähig zur Reform?, in: Wolf, K.-D. (Hg.): Staat und Gesellschaft - fähig zur Reform? 23. wissenschaftlicher Kongress der Deutschen Vereinigung für Politische Wissenschaft, Baden-Baden, 2007, 19-33, hier 25.

25 Schmidt, M. G.: Wörterbuch zur Politik, 2. Aufl., Stuttgart, 2004, 597.

26 Davon unberührt bleibt die Tatsache, dass auch Reformen langfristig revolutionäre Wirkungen entfalten können.

27 Krockow, C. Graf v.: Reform als politisches Prinzip, München, 1976, 12.

28 Tsebelis, G.: Nested Games. Rational Choice in Comparative Politics, Berkeley u. a., 1990, 104.

29 Vgl. zum weiteren Kontext: Benoit, K.: Electoral Laws as Political Consequences: Explaining the Origins and Change of Electoral Institutions, in: Annual Review of Political Science, 10 (2007), 363390 . 
Interessant ist im Weiteren nicht nur die Abgrenzung zwischen wie auch immer verursachtem Wandel einerseits und gezielten politischen Reformen des Staates andererseits, sondern auch das Verhältnis zwischen Reform und Wandel: Auf den ersten Blick scheint es, als wenn Reformen gleichsam naturgemäß ein progressiver Zug eigen sei. Das ist jedenfalls gewiss die dominante Konnotation des Reformbegriffs. Dies erweist sich bei näherer Betrachtung jedoch als fragliche Annahme. So lässt sich kaum bestreiten, dass es neben progressiven Reformen auch solche Reformen gibt, die von ihrem grundsätzlichen Motiv her primär auf die Bewahrung des Bestehenden gerichtet sind und in diesem Sinne einen eindeutig konservativen Charakter besitzen. Die politisch entscheidende Frage ist dann freilich, ob es bei Reformen der konservativen Kategorie um die Verteidigung von Privilegien oder hingegen um das Eintreten für die Bewahrung bestimmter etablierter normativer Standards der liberalen Demokratie unter veränderten politisch-gesellschaftlichen Bedingungen geht.

Beispiele für Reformen, die von dem Bestreben politischer Akteure getragen sind, ihre eigene Machtposition zu bewahren, finden sich gehäuft auf dem Feld der Parteienfinanzierung, deren Ziel darin bestehen kann, lästige Herausforderer durch die Institutionalisierung ungleicher Wettbewerbsbedingungen auf Abstand zu halten. Diese Gefahr besteht keineswegs nur in strukturell defekten oder jungen Demokratien, sondern auch in konsolidierten, „reifen“ Demokratien. In Deutschland hat es aus diesem Grunde nicht zufällig zahlreiche Interventionen des Bundesverfassungsgerichts gegeben, mit denen Korrekturen an den geschaffenen Regeln eingefordert wurden. ${ }^{30}$

Eine von ihrer politischen Stoßrichtung her vollständig andere Gruppe von „,konservativen" Reformen versammelt Maßnahmen, mit denen versucht wird, etablierte normative Standards der Demokratie an veränderte gesellschaftliche Erwartungen bzw. Bedingungen anzupassen - auch dann, wenn im Zuge dessen die Machtposition bislang dominierender Akteure gegebenenfalls zur Disposition gestellt wird.

30 Vgl. aus politikwissenschaftlicher Perspektive Landfried, C.: Parteifinanzen und politische Macht, Baden-Baden, 1994, 78-84; Vanberg, G.: The Politics of Constitutional Review in Germany, Cambridge, 2005, 143-167. - Die Befunde der jüngeren empirischen Forschung deuten jedoch darauf hin, dass es in den konsolidierten Demokratien Europas insgesamt nicht zu einer ,Versteinerung des Parteienwettbewerbs" durch Reformen der Parteienfinanzierung gekommen ist. Vgl. etwa Naßmacher, $H$.: Parteiensysteme und Parteienfinanzierung in Westeuropa, in: Niedermayer, O./Stöss, R./Haas, M. (Hg.): Die Parteiensysteme Westeuropas, Wiesbaden, 2006, 507-519; Scarrow, S.: Party Subsidies and the Freezing of Party Competition: Do Cartel Mechanisms Work?, in: West European Politics, 29/4 (2006), 619-639. 
Eine Entscheidung darüber, ob besser von konservativen oder progressiven Reformen zu sprechen wäre, fällt gelegentlich schwer: Das zeigt etwa die Auseinandersetzung über das Wahlrecht für Migranten: Aus konservativer Warte erscheint die mögliche Gewährung des Wahlrechts an Personen ohne die Staatsangehörigkeit des betreffenden Landes als geradezu radikal-progressiv. Sie droht gleichsam, das Prinzip der Volkssouveränität auszuhebeln. Daneben gibt es freilich eine alternative Lesart der Problemkonstellation: Bei ihr erscheint die Gewährung des Wahlrechts an ausländische Personen, die dauerhaft in dem betreffenden Land leben, über ihre Sprachkompetenz die Mindestvoraussetzung für eine Teilnahme am gesellschaftlichen Diskurs besitzen und über Steuern und Abgaben möglicherweise auch die Leistungen des Staates mitfinanzieren, lediglich als zeitgemäße Adaption eines traditionellen Kernprinzips der repräsentativen Demokratie, demzufolge die Regierten das Recht besitzen (sollen), die Regierenden aus- und abzuwählen. ${ }^{31}$

\section{Demokratiereformen als spezifische Ausprägung politischer Reformen}

Die Grenzen zwischen Reformen in der Demokratie und Reformen an der Demokratie sind insoweit fließend, als sich Reformen an der Demokratie ebenfalls im Kontext demokratischer Strukturen vollziehen. Demokratiereformen zielen dabei auf die polity- und die politics-Dimension der Demokratie, auf die Veränderung der Strukturen demokratischer Gemeinwesen und der in ihnen ablaufenden Prozesse. Allerdings wäre es unsinnig, Demokratiereformen, mit denen auf diese beiden Dimensionen gestaltend Einfluss zu nehmen versucht wird, eine policy-Dimension abzusprechen. Aus einer policy-Perspektive erscheinen Demokratiereformen als das Ergebnis von „Demokratiepolitik ${ }^{\text {‘32. }}$.

31 Pelinka, A.: Demokratie - Weg und Ziel. Zwischen Gleichheit und Differenz, in: Wiener Hefte zu Migration in Theorie und Praxis, 1/1 (2003), 45-53. In den allermeisten Ländern existieren heute Wahlrechtsregime, die auf das Prinzip des ,ethnischen Nationalismus“ gegründet sind. Dabei besitzen Staatsbürger (seien sie im Staatsgebiet ansässig oder auch nicht) das Wahlrecht. Zumindest in der Theorie gibt es sowohl restriktivere als auch expansivere Wahlrechtsregime: Das republikanische Modell sieht ein Wahlrecht nur für Staatsbürger vor, die in dem betreffenden Staat leben - also ein restriktiveres Modell. Andere Lösungsversuche möchten das Wahlrecht all jenen zugestehen, die von einer politischen Entscheidung der Regierung potentiell betroffen sind - also möglicherweise auch Personen, die weder die Staatsangehörigkeit des Landes besitzen noch in diesem Land leben; vgl. Bauböck, R.: Expansive Citizenship - Voting beyond Territory and Membership, in: PS: Political Science \& Politics, 38/4 (2005), 683-687.

32 Greven, M.Th.: Sind Demokratien reformierbar? Bedarf, Bedingungen und normative Orientierungen für eine Demokratiereform, in: Offe, C. (Hg.), a. a. O., 72-91, hier 76. 
Zugegeben: der Begriff „Demokratiepolitik“ ist nicht sehr gebräuchlich, jedenfalls gewiss weniger verbreitet als jener der Verfassungspolitik bzw. Verfassungsreformpolitik, der vielfach als Synonym für Reformen an der Demokratie benutzt wird. Mit Blick auf die inhaltliche Treffsicherheit erscheint es gleichwohl gerechtfertigt, dem Begriff „Demokratiepolitik“ den Vorzug zu geben, denn tatsächlich können Demokratiereformen an sehr unterschiedlichen Punkten ansetzen - keineswegs ausschließlich an den verfassungsrechtlich kodifizierten Strukturen. ${ }^{33}$

Historisch war die Unterscheidung zwischen „Demokratiereformen“ und Akten der "Demokratisierung“ in den demokratischen Pionierregimen gleichsam notwendiger Weise wenig trennscharf: Eine Demokratisierung bestehender, bereits teildemokratisierter Strukturen wurde durch Reformen angestrebt, die weithin als „Demokratiereformen“ wahrgenommen wurden - etwa die großen britischen Wahlrechtsreformen aus dem letzten Drittel des 19. Jahrhunderts. Dies erklärt sich daraus, dass es zunächst praktisch keine höheren empirischen Standards der modernen liberalen Demokratie als die jeweils erreichten gab. Erst von der nächsten Stufe der Demokratisierung aus, im historischen Rückblick betrachtet, wurden die demokratiebezogenen Defizite des status quo ante in ganzer Schärfe deutlich.

Die jüngere empirisch-vergleichende Demokratieforschung hat die klassische Gegenüberstellung demokratischer und nicht-demokratischer Regime weitgehend aufgegeben zugunsten einer vergleichenden Analyse der Demokratiequalität unterschiedlicher (mehr oder minder) demokratischer Regime. Damit sind auch die Grenzen zwischen Prozessen der Demokratisierung, durch die eine demokratische Ordnung zunächst einmal geschaffen werden muss, und Demokratiereformen durchlässiger geworden. Ein Ausdruck dieser paradigmatischen Verschiebung ist die Einsicht, dass auch mit Demokratiereformen, die diese Bezeichnung mit Blick auf ihren strukturellen Kontext eindeutig rechtfertigen, möglicherweise ein Mehr an Demokratie erstrebt wird - eine Agenda, ein Pro-

33 Wichtige konzeptuelle Perspektiven und analytische Einsichten der jüngeren politikwissenschaftlichen Forschung zum hier nicht näher behandelten Gegenstand der Verfassungsreform finden sich etwa bei Behnke, N./Benz, A.: The Politics of Constitutional Change between Reform and Revolution, in: Publius, 39/2 (2009), 213-240; Abromeit, H.: Was begünstigt und was behindert Verfassungsreformen?, in: Wolf, K.-D. (Hg.), a. a. O., 59-76; Köppl, S.: Zur vergleichenden Analyse von Verfassungsreformprozessen. Konzeptuelle Überlegungen, in: Wolf, K.-D. (Hg), a.a. O., 77-95; Lorenz, A.: Stabile Verfassungen? Konstitutionelle Reformen in Demokratien, in: Zeitschrift für Parlamentsfragen, $35 / 3$ (2004), 448-468; Schultze, R.-O.: Verfassungsreform als Prozess, in: Zeitschrift für Parlamentsfragen, 28/3 (1997), 502-520. 
zess, den man treffend als „Demokratisierung der Demokratie“34 ${ }^{\text {(34 }}$ bezeichnen kann.

Auf den ersten Blick scheint diese Formel aus sich selbst heraus verständlich. Aus dem eingangs umrissenen Demokratieverständnis ergibt sich indes, dass Demokratiereformen zwar auf eine Erweiterung demokratischer Partizipationsoptionen gerichtet sein können (womit gleichsam der Kernbereich der Demokratisierung im engeren Wortsinn bezeichnet wäre), aber keineswegs notwendigerweise auf diesen Aspekt beschränkt sind. Reformbestrebungen können sich vielmehr ebenso gut auf andere Ziele - wie die Erhöhung von Transparenz oder die Stärkung der Effektivität der Herrschaftsausübung - richten. Dem übergeordneten Ziel einer als komplexes Unternehmen verstandenen „Demokratisierung der Demokratie“ kann gegebenenfalls sogar durch Maßnahmen wie die Einrichtung oder Aufwertung „nicht-demokratischer“, „nicht-majoritärer Institutionen“ - wie insbesondere der Verfassungsgerichtsbarkeit - entsprochen werden. In Teilen der jüngeren Literatur, so bei Kim Lane Scheppele, wurde gar die These vertreten, dass Verfassungsgerichte von ihren Handlungen her im Kern demokratischer seien als Parlamente und dass ihre Präsenz und ihr Handeln die politischen Eliten des Systems demokratischer machten. ${ }^{35}$ In dieselbe Richtung schlagen Bewertungen, die Verfassungsgerichten die Fähigkeit zur erfolgreichen Erziehung der Gesellschaft zur Demokratie attestieren. ${ }^{36}$ Die dominante positive Bewertung der Verfassungsgerichtsbarkeit im jüngeren akademischen Diskurs indiziert ohne Zweifel einen grundlegenden Wandel des vorherrschenden Verständnisses liberaler Demokratie, der wegführt von einer Überbewertung der rein elektoralen Dimension demokratischer Gemeinwesen: „Today, constitutional

34 Offe, C. (Hg.): Demokratisierung der Demokratie, a. a.O.

35 Scheppele, K.L.: Democracy by Judiciary, Or why Courts Can be More Democratic than Parliaments, in: Czarnota, A./Krygier, M./Sadurski, W. (Hg.): Rethinking the Rule of Law after Communism, Budapest, 2005, 25-60.

36 Bryde, B.-O.: Die Rolle der Verfassungsgerichtsbarkeit in Umbruchsituationen, in: Hesse, J.J./Schuppert, G.F./Harms, K. (Hg.): Verfassungsrecht und Verfassungspolitik in Umbruchsituationen. Zur Rolle des Rechts in staatlichen Transformationsprozessen in Europa, Baden-Baden, 1999, 197-210, hier 201. Freilich gibt es auch kritischere Stimmen wie von Wojciech Sadurski, der mit Blick auf die jungen Demokratien Ostmitteleuropas eher einen negativen Effekt der Verfassungsgerichte dieser Region auf die demokratische Beteiligung und den öffentlichen demokratischen Diskurs über gesellschaftlich umstrittene Themen erkennt; vgl. Sadurski, W: Rights Before Courts: A Study of Constitutional Courts in Postcommunist States of Central and Eastern Europe, Dordrecht u. a., 2005. 
democracy is defined in terms of the new constitutionalism, which assumes that constraining majority rule with rights and review is a good thing. “" ${ }^{37}$

\section{Kernaspekte und Erkenntnisziele des Studiums der „Demokratisierung der Demokratie“"}

Betreiber und Betrachter von Demokratiereformen sind gleichermaßen gut beraten, ihr Augenmerk zunächst auf die institutionellen Arrangements demokratischer Regime zu richten. Und sei es lediglich deshalb, weil Institutionen in der Tat jenen potentiell veränderbaren Faktor in einem Meer an kaum oder ungleich schwerer zu verändernden Faktoren darstellen, die den Charakter eines Gemeinwesens nachhaltig prägen. ${ }^{38}$ Aber auch über den Einfluss von Institutionen auf demokratische Politik in ihren unterschiedlichen Dimensionen besteht heute weniger Zweifel denn je. Die gewachsene Unterstützung für die „Institutions Do Matter! "-Vermutung erklärt sich dabei zu nicht geringen Teilen aus dem Wandel des Institutionenverständnisses selbst: Zeitgemäße Institutionenbegriffe umspannen geradezu selbstverständlich nicht nur die formalen, sondern zugleich die informalen Institutionen. ${ }^{39}$

Die ausdrückliche Berücksichtigung informaler Institutionen löst auch das (zum Teil künstlich befestigte) Spannungsverhältnis zwischen Institutionen und Kultur auf, das die ältere Debatte über politische Institutionen prägte, insbesondere im Zusammenhang mit Erklärungsversuchen gescheiterter Exportversuche politischer Institutionen. ${ }^{40}$ Selbst in demokratiefeindlichen kulturellen Kontexten kommt es in den seltensten Fällen zur vollständigen Abschaffung der formalen institutionellen Arrangements, sondern eher zur Konstituierung informaler Institutionen, durch die die formalen Institutionen der Demokratie gegebenenfalls ergänzt, überlagert oder faktisch außer Kraft gesetzt werden. ${ }^{41}$

37 So Stone Sweet, A.: Constitutions and Judicial Power, in: Caramani, D. (Hg.): Comparative Politics, Oxford, 2008, 217-239, hier 235.

38 Rockman, B.A.: The Performance of Presidents and Prime Ministers and of Presidential and Parliamentary Systems, in: von Mettenheim, K. (Hg.): Presidential Institutions and Democratic Politics. Comparing Regional and National Contexts, Baltimore/London, 1997, 45-64, hier 64.

39 Rhodes, R.A.W./Binder, S.A./Rockmann, B.A. (Hg.): The Oxford Handbook of Political Institutions, Oxford, 2006, 3-20.

40 Przeworski, A.: Institutions Matter?, in: Government and Opposition, 39/4 (2004), 527-540.

41 Lauth, H.-J.: Informal Institutions and Democracy, in: Democratization, 7/1 (2000), 7-50; Helmke, G./Levitsky, S.: Informal Institutions and Comparative Politics: A Research Agenda, in: Perspectives on Politics, 2/4 (2004), 725-739; dies. (Hg.): Informal Institutions and Democracy. Lessons from Latin America, Baltimore/London, 2006. 
Zum Credo des Neo-Institutionalismus gehört jedoch nicht nur ein breiteres und dynamisiertes Verständnis von Institutionen, aus denen politischen Akteuren spezifische „opportunities“ und „constraints" erwachsen, sondern auch die Überzeugung, dass Akteure die Möglichkeit besitzen, die sie umgebenden institutionellen Arrangements zu verändern. Ohne die konzeptuelle Anerkennung dieser Interdependenz von Institution und Akteur wäre das Studium von Bestrebungen institutioneller Demokratiereformen nicht nur sinnlos, sondern auch unmöglich.

Auf der Forschungsagenda von Demokratiereformstudien stehen deshalb die zentralen verfassungsrechtlichen und weiteren formal-institutionellen Parameter liberal-demokratischer Systeme ebenso wie die weicheren informalen Institutionen, die demokratischen Regimen in der Verfassungspraxis ihr spezifisches Gepräge verleihen. Überkommenen Vorurteilen gegenüber institutionalistischen Ansätzen entgegen bleibt die komplexe gesellschaftliche Dimension der Demokratie bei einem solchen konzeptuellen Zugriff keineswegs außen vor.

Mindestens drei im weiteren Sinne gesellschaftsbezogene Aspekte lassen sich unterscheiden, auf die im Rahmen einer Analyse von Reformen an der Demokratie achtzugeben ist: Erstens kann die Gesellschaft als zentraler Referenzakteur und Rezipient staatlicher Reformagenden bzw. Reformen des Staates gelten. Auseinandersetzungen über Demokratiereformen finden nicht nur in einem bestimmten politischen Kräftefeld und unter bestimmten institutionellen Bedingungen statt, sondern zugleich in einem spezifischen gesellschaftlich-kulturellen Kontext. Man könnte die gesellschaftlichen Werthaltungen auch als die politischkulturellen Parameter von Reformen an den Institutionen der Demokratie bezeichnen. Als das vielleicht wichtigste Kriterium von Demokratiereformen aus Sicht der Gesellschaft kann die Frage gelten, ob mit ihnen die Legitimität im Sinne der „Anerkennungswürdigkeit“ der betreffenden Ordnung vergrößert wird oder nicht. Im Gegensatz zu anderen Verständnissen von Legitimität, die auf die „Rechtmäßigkeit“ einer Ordnung konzentriert sind, stellt ein auf die Anerkennungswürdigkeit bezogener Legitimitätsbegriff gleichsam eine Verknüpfung zwischen der staatlichen Ordnung und der Gesellschaft her. Aus dieser Perspektive betrachtet können eine staatliche Ordnung und die in ihr ablaufenden Willensbildungs- und Entscheidungsprozesse niemals ,aus sich selbst heraus“ als legitim gelten, wie auch immer ihre Strukturen beschaffen sein mögen. ${ }^{42}$

42 Gerade daraus können sich im Vergleich unterschiedlicher Regime, speziell älterer und jüngerer Demokratien, erhebliche Bewertungsprobleme ergeben. Wie Studien etwa über die jungen Demokratien Ost- 
Zweitens interessiert die Gesellschaft als möglicher Impulsgeber von Demokratiereformen. Reformpolitische Impulse aus der Gesellschaft sind keineswegs ausschließlich über die repräsentativ-demokratischen Strukturen und Kanäle, sondern auch auf der Grundlage unkonventioneller Beteiligung sowie gegebenenfalls direktdemokratischer Mitsprache möglich. Insofern ist Feststellungen zu widersprechen, denen zufolge ,der Motor jeder Demokratiereform [...] die Regierung" sei. ${ }^{43}$ Abgesehen von den empirischen Verkürzungen einer solchen Sichtweise vermögen auch deren demokratietheoretischen Implikationen nicht recht zu überzeugen. Mit Blick auf ihr legitimitätsstiftendes Potential wären reformpolitische Maßnahmen, die ihre Entstehung und Umsetzung tatsächlich allein der Regierung verdanken, zumindest als „,suboptimal“ zu klassifizieren.

Drittens schließlich muss sich jeder wissenschaftliche Betrachter demokratischer Reformprozesse - wie jeder Demokratiereformer selbst - auch und besonders deshalb für die Gesellschaft interessieren, als es bekanntlich keine „Demokratie ohne Demokraten“ gibt. Diese Feststellung ist im hier entwickelten Kontext nicht nur von konzeptueller, sondern auch von gegenständlich-inhaltlicher Relevanz: So kann, neben den unterschiedlichen institutionellen Reformen, auch die „Demokratieerziehung“, die politische Bildung als ein zentraler Gegenstand bzw. eine zentrale Strategie der „Demokratisierung der Demokratie“ gelten.

Die politische Bildung verkörpert im Rahmen eines komplexeren Modells der Bedingungen einer nachhaltigen Demokratieentwicklung, bei der es zunächst um die Entlastung der unterschiedlichen Lehrinstitutionen von den Zwängen politischer Macht und ökonomischer Interessen geht ${ }^{44}$, gleichsam die zweite, positive Ebene. Wie kaum anders zu erwarten, ist der negative Konsens über potentielle Strukturdefizite des Bereichs größer als die Übereinstimmung über ein angemessenes Programm der politischen Bildung. Gleichwohl gibt es grundlegende Gemeinsamkeiten zwischen unterschiedlichen Positionen. Eine Beschränkung der Demokratieerziehung auf die bloße Vermittlung eines Verständnisses der Funktionsvoraussetzungen der Staatsorgane wird heute zu Recht als unzureichend

europas zeigen, gibt es in post-sozialistischen Kontexten ein hohes Potential an Anerkennungsbereitschaft auch gegenüber einigen solcher informaler Institutionen und Praktiken, die mit den normativen Grundprinzipien der liberalen Demokratie westlicher Prägung allenfalls zum Teil vereinbar erscheinen; vgl. Steinsdorff, S. v.: Gute und schlechte Informalität? Informelle Politik in West und Ost, in: Osteuropa, 55/10 (2005), 5-15.

43 Neisser, H., a. a. O., 428.

44 Preuß, U.K.: Die Bedeutung kognitiver und moralischer Lernfähigkeit für die Demokratie, in: Offe, C. (Hg.), a. a. O., 259-280. 
abgelehnt. Ebenfalls weitgehend konsensfähig ist die Position, dass die Demokratieerziehung sich neben der „Demokratie als Herrschaftsform“ auch auf die beiden komplementären Dimensionen der Demokratie als "Gesellschaftsform“ und „Lebensform“ "erstrecken muss. ${ }^{45}$

Umstritten bleibt unter anderem die Frage, wie stark im Rahmen entsprechender Bemühungen der Aspekt der Partizipation betont werden sollte. Als eigentliches Herzstück der Demokratie steht die Wünschbarkeit einer großen Beteiligung nicht wirklich zur Debatte; sie verdient gleichsam als ein Wert an sich betrachtet $\mathrm{zu}$ werden. In einigen jüngeren Beiträgen wurde angeregt, die Bereitschaft der Bürger zur Partizipation an demokratischen Verfahren mit dem Hinweis zu vergrößern, dass politische Beteiligung „echte Wirksamkeit“ bedeuten könne. ${ }^{46}$ Das erscheint sympathisch und kühn zugleich. In welchem Maße solche Verheißungen auch für zunehmend internationalisierte Entscheidungskontexte und -prozesse Geltung beanspruchen können, wird sich erweisen müssen. Jedenfalls ist skeptischeren Beobachtern, die die Entwicklung eines neuen Enttäuschungspotentials befürchten, das sich aus bewusst geförderten (möglicherweise überhöhten) Erwartungen von Bürgern an die Demokratie speisen könnte ${ }^{47}$, nicht schlicht eine mangelnde Begeisterung für die Demokratie zu unterstellen.

Das Studium nicht-institutioneller Aspekte von Demokratiereformen darf sich jedoch nicht auf die Ebene der Bürger beschränken. Hinzu kommen muss die Beschäftigung mit den Inhabern politischer Ämter, den Repräsentanten der Gesellschaft, ihrer Befähigung und Bereitschaft, den Anforderungen liberaler Demokratie gerecht zu werden. Dabei kann es nicht lediglich um bestimmte handwerkliche Fertigkeiten von Amtsinhabern gehen. Ebenfalls zu diskutieren sind die wünschenswerten moralischen Eigenschaften von Inhabern politischer Ämter und die Bedingungen, denen ein begünstigender Einfluss auf die Erreichung normativ erstrebter Zielsetzungen zugetraut werden kann.

In der einschlägigen Literatur finden sich zahlreiche prinzipielle Vorbehalte, die eine Beschäftigung mit „Politikertugenden“ als kaum zu rechtfertigendes Unter-

45 Himmelmann, G.: Durch Demokratie-Lernen zum Demokratiebewusstsein, in: Lange, D./Himmelmann, G. (Hg.): Demokratiebewusstsein. Interdisziplinäre Annäherungen an ein zentrales Thema der Politischen Bildung, Wiesbaden, 2007, 26-40.

46 Leggewie, C./Schneider, J.: Demokratie in Gefahr? Wie man Beteiligungskompetenz zurückerobert, in: Gesellschaft - Wirtschaft - Politik, 57/3 (2008), 383-396, hier 389.

47 Patzelt, W.J.: Was für eine Art von „Demokratieerziehung“ brauchen wir?, in: Gesellschaft - Wirtschaft - Politik, 56/3 (2007), 343-354. 
nehmen erscheinen lassen. ${ }^{48}$ Die unterschiedlichen Vorbehalte bzw. Einwände lassen sich jedoch jeder für sich ohne unverhältnismäßigen Aufwand entkräften: Der radikaldemokratische Einwand - der sich bereits an der Unterscheidung zwischen Regierenden und Regierten, zwischen Repräsentanten und Repräsentierten stört und deshalb eine Diskussion über die wünschenswerten Eigenschaften politischer Amtsinhaber von vornherein ablehnt - übersieht, dass die moderne Demokratie ohne eine solche Arbeitsteilung nicht auskommt. Allerdings kann zu den Vorteilen der repräsentativen gegenüber der identitären Demokratie kaum eine Entlastung der Bürger in ihrer Verantwortung für das Gemeinwesen gezählt werden: Die gemeinsame Verantwortung von Repräsentanten und Repräsentierten ist in der repräsentativen Demokratie lediglich institutionell differenziert, keineswegs substantiell aufgehoben. ${ }^{49}$

Eine zweite Position - die „systemtheoretische Position“, die angesichts der Komplexität moderner politischer Systeme praktisch keinen Handlungsspielraum individueller Amtsinhaber sieht und aus diesem Grunde das Thema für nichtig erachtet - unterschätzt den „Faktor Mensch“. Letzten Endes hängt alles Handeln des Staates auch unter den Bedingungen der modernen liberalen Demokratie an Menschen. ,Government is human beings acting “, wie ein amerikanischer Autor diesen Sachverhalt elegant auf den Punkt brachte. ${ }^{50}$ Etwas anderes kommt hinzu: Wenn man den gängigen Interpretationen der jüngeren Wandlungstendenzen in den konsolidierten liberalen Demokratien folgt, hat sich das Eigengewicht der einzelnen Persönlichkeit in der Politik sogar erhöht. Das ist jedenfalls der Befund von Arbeiten, die einen tief greifenden und anhaltenden Trend in Richtung „Personalisierung“ ausmachen; ${ }^{51}$ einigen Betrachtern erscheint „Personalisierung“ gar als das vielleicht wichtigste Merkmal demokratischer Politik im 21. Jahrhundert überhaupt. ${ }^{52}$

48 Vgl. Rinderle, P.: Welche moralischen Tugenden braucht der Politiker in der liberalen Demokratie?; in: Zeitschrift für Politik, 50/4 (2003), 397-422; ders.: Gerechtigkeit, Gemeinwohl und prozedurale Integrität, in: Zimmer, A./Jankowitsch, R. (Hg.): Political Leadership. Annäherungen aus Wissenschaft und Praxis, Berlin u. a., 2008, 235-260.

49 Pfiffner, J.P.: The Paradox of Governmental Power, in: Kennedy, M./Hoxie, R. G./Repland, B. (Hg.): The Moral Authority of Government, New Brunswick/London, 2000, 183-188.

50 Harriss, C.L.: The Moral Authority of Government, in: Kennedy, M./Hoxie, R. G./Repland, B. (Hg.), a. a. O., 113-117, hier 115 .

51 Caprara, G. V.: The Personalization of Modern Politics, in: European Review, 15/2 (2007), 151-164.

52 McAllister, I.: The Personalization of Politics, in: Dalton, R.J./Klingemann, H.-D. (Hg.): The Oxford Handbook of Political Behavior, Oxford, 2007, 571-588, hier 585. 
Die dritte Position, die man als die „realpolitische“ Position bezeichnen könnte, bestreitet den Sinn der Frage nach speziellen Tugenden politischer Amtsinhaber mit dem Hinweis, dass der Bereich staatlicher Politik, von politischer Machtausübung überhaupt für die Anwendung moralischer Standards nicht geeignet sei. Richtig daran ist vermutlich, dass Aspekte privater Moral auf der Ebene öffentlicher Ämter und Amtsführung möglicherweise nicht wirklich die gleiche Geltung beanspruchen können wie im Privatleben. Einschlägige Studien weisen jedenfalls darauf hin, dass es keinen systematischen Zusammenhang zwischen den moralischen Standards im Privatleben von politischen Amtsinhabern einerseits und deren politischen Effektivität im Amt andererseits gibt. Das Bedürfnis, die Wahrheit zu sagen, erscheint kontext- und gegenstandsbezogen. ${ }^{53}$ Daraus wurde im Rahmen generellerer Erwägungen gefolgert, dass eine Betrachtung und Bewertung politischer Amtsinhaber durch eine Brille „privater Moralität“ den Ansprüchen der Führung öffentlicher Ämter generell nicht gerecht werde. Der geeignete Maßstab für die Bewertung der Führung öffentlicher Amtsgeschäfte sei nicht ,private morality“, sondern ,public virtue“ - wozu unter anderem politische Umsicht, das Eintreten für Gerechtigkeit und Frieden und dergleichen mehr gezählt werden könnten. ${ }^{54}$

Wichtig zu sehen ist bei alledem, dass die Zurückweisung privater moralischer Standards als Bewertungsgrundlage für öffentliches politisches Handeln die Forderung nach spezifischen persönlichkeitsbezogenen Voraussetzungen für die Übernahme eines politischen Amtes nicht ausschließt. Im Zentrum entsprechender Diskussionen, steht das schillernde Konzept der „Integrität“. Peter Rinderle betont dabei eine Reihe unterschiedlicher Aspekte: ${ }^{55}$ Grundlegend für das Konzept der „Integrität“ ist zunächst die Übereinstimmung von Werten und Handeln; Integrität kann Personen zugeschrieben werden, die so handeln, wie ihre Werte es ihnen vorschreiben; sie sind jeweils selbst Autor ihrer Handlungen. Zweitens nennt Rinderle den Aspekt der Übereinstimmung von Handlungsgründen und Handlungsmotiven: Ein integrer Mensch handelt nicht etwa nur aufgrund zufälliger äußerer Umstände, sondern lässt sich bei der Handlungsmotivation von seinen Gründen selbst leiten; er handelt aufgrund seiner besten Handlungsgründe. Drittens haben sich die Werte und Prinzipien, die praktischen Urteile, an

53 Solomon, R. C.: Is It Ever Right To Lie? The Philosophy of Deception, in: The Chronicle of Higher Education, 27.02.1998, A60.

54 Harriss, C.L., a.a. O., 117.

55 Rinderle, $P$., a.a. O. 
denen die integere Person ihr Handeln ausrichtet, dadurch auszuzeichnen, dass sie einer rationalen Deliberation, einer öffentlichen beratenden Auseinandersetzung standzuhalten vermögen.

In der politischen Praxis liberaler Demokratien wird die Tugend des Politikers vor allem in der Bereitschaft zu sehen sein, Verantwortung für die Integrität politischer Institutionen zu übernehmen. Im Kern geht es dabei um die Entwicklung und Aufrechterhaltung eines „Amtsethos“. Als Amtsinhaber hat der Politiker die Integrität des Amtes im Gefüge der politischen Institutionen zu schützen; zugleich trägt er eine Mitverantwortung auch für das Verhalten seiner Kollegen, denen grundsätzlich ein ähnlicher Auftrag aufgegeben ist wie ihm selbst. Gelegentlich wurde gar eine moralische Verpflichtung politischer Amtsinhaber postuliert, im Bedarfsfall mit psychologisch-erzieherischen Maßnahmen gegenüber den Bürgern das Wohl der liberalen Demokratie zu verteidigen, die Gesellschaft gleichsam zur Demokratie zu erziehen. Das Spannungsverhältnis zwischen dieser Forderung und den Prämissen der repräsentativen Demokratie ist unmittelbar greifbar, aber selbst die Erfüllung der vergleichsweise bescheideneren Erwartungen kann in der Praxis keineswegs als selbstverständlich vorausgesetzt werden.

Skandale und Krisen, die von breiter öffentlicher Empörung über das Verhalten politischer Entscheidungsträger und moralischen Appellen an die politische Klasse begleitet werden, fordern die Verantwortlichen regelmäßig zu demonstrativen Gesten der „Selbstläuterung“ heraus. Die liberale Demokratie, deren Errungenschaft nicht zum letzten darin besteht, dass die Chance eines angemessenen Verhaltens von politischen Machthabern nicht mehr allein auf den Glücksfall persönlicher Tugendhaftigkeit und Charakterstärke der Betreffenden angewiesen ist, kann und muss sich mit Aktionen freiwilliger Selbstkontrolle jedoch nicht begnügen. Der moralischen Selbsterziehung der politischen Elite kommt im Rahmen eines deutlich breiter angelegten Kontrollregimes lediglich der Stellenwert einer ergänzenden Komponente zu. Zeitgemäße Konzepte politischer Kontrolle umfassen zahlreiche politische und rechtliche Komponenten, die auf unterschiedlichen zeitlichen Ebenen wirksam werden. ${ }^{56}$ Von nicht zu unterschätzender Bedeutung bleiben auch in einem Entwicklungsstadium der liberalen Demokratie, das gelegentlich als ,post-parlamentarisch“ beschrieben wurde, die

56 Mulgan, R.: Holding Power to Account. Accountability in Modern Democracies, Basingstoke, 2003; Helms, L.: The Changing Parameters of Political Control in Western Europe, in: Parliamentary Affairs, 59/1 (2006), 78-97. 
unterschiedlichen Ausprägungen parlamentarischer Kontrolle. ${ }^{57}$ Diese wird in ihren Wirkungen verstärkt durch die politische Berichterstattung unabhängiger Massenmedien. Die Medien haben jedoch längst nicht mehr allein die Rolle eines bloßen Transmitters politischer Entwicklungen in der parlamentarischen Arena inne, sondern verfolgen das politische Treiben mit einem Interesse, das nur noch zum Teil im Dienste einer dem Gemeinwesen verpflichteten politischen Kontrolle steht. Dieser Anspruch wird zumindest mit handfesten kommerziellen Interessen vermengt, die eine Kultur der "Skandalisierung“ begünstigen, von der auch das Ansehen redlicher Amtsinhaber beschädigt zu werden droht und welche geeignete Kandidaten möglicherweise gar bereits von einem Einstieg in die Politik zurückscheuen lässt. ${ }^{58}$ Die gerichtliche Kontrolle des Regierungshandelns und des Verhaltens politischer Amtsinhaber hat sich in den meisten konsolidierten liberalen Demokratien in den vergangenen Jahrzehnten deutlich intensiviert. ${ }^{59}$ Die schärfste demokratische Waffe im Arsenal der liberalen Demokratie bleibt jedoch die politische Kontrollmacht des Wählers.

An dieser Stelle schließt sich der Kreis, wird der Bezug zwischen weiter reichenden normativen Agenden der Demokratiereform und vergleichsweise unspektakulären Reformen des Institutionensystems unmittelbar greifbar: Eine effektive Wahrnehmung der Kontrollfunktion der Wähler gegenüber der Regierungselite setzt das Vermögen voraus, missliebige „Regenten“ bei Bedarf aus dem Amt zu befördern. Die Erfahrung zeigt zwar, dass kein noch so ausgeklügeltes ,,institutional engineering “ alle Unwägbarkeiten der Demokratie im Voraus zu kalkulieren vermag, aber speziell die Fähigkeit von Wahlrechts- und Wahlsystemreformen, die Bedingungen politischen Wettbewerbs und elektoraler Kontrolle nachhaltig in bestimmter Richtung zu beeinflussen, steht weitgehend außer Frage. ${ }^{60}$ An ihre Grenzen gelangt die demokratische Methode in Kontexten, in denen nicht nur die Regierungselite, sondern auch weite Teile der oppositionel-

57 Holtmann, E./Patzelt, W.J. (Hg.): Kampf der Gewalten? Parlamentarische Regierungskontrolle - gouvernmentale Parlamentskontrolle: Theorie und Empirie, Wiesbaden, 2004; Bußjäger, P. (Hg.): Die Zukunft der parlamentarischen Kontrolle, Wien, 2008.

58 Helms, L.: Governing in the Media Age: The Impact of the Mass Media on Executive Leadership in Contemporary Democracies, in: Government and Opposition, 43/1 (2008), 26-54.

59 Kühn, Z.: The Judicialization of European Politics, in: Heywood, P. u. a. (Hg.): Developments in European Politics, London, 2006, 216-236; Hirschl, R.: The Judicialization of Mega-Politics and the Rise of Political Courts, in: Annual Review of Political Science, 11 (2008), 93-118.

60 Lijphart, A.: Electoral Systems and Party Systems, Oxford, 1994; Nohlen, D.: Wahlrecht und Parteiensystem, 5. Aufl., Opladen, 2007; Baedermann, T.: Der Einfluss des Wahlrechts auf das Parteiensystem, Baden-Baden, 2007; Papparlardo, A.: Electoral Systems, Party Systems: Lijphart and Beyond, in: Party Politics, 13/6 (2007), 721-740. 
len „Alternativregierung“ des politischen Vertrauens unwürdig sind oder aber die kompetitive Dimension von Wahlen durch Absprachen zwischen koalierenden Parteieliten faktisch ausgehebelt wird. ${ }^{61}$ Als umso wertvoller können sich unter diesen Bedingungen direktdemokratische Beteiligungsrechte des Volkes erweisen, mit denen zwar keine personellen, aber sehr wohl wichtige policy-bezogene Kontrollwirkungen erzeugt werden können. ${ }^{62}$

\section{Statt eines Fazits: Probleme und Potentiale der „Demokratisierung der Demokratie“"}

Die Probleme von Demokratiereformen wie ihrer staats- und politikwissenschaftlichen Analyse sind vielfältig. Zu den greifbarsten Problemen in der politischen Arena gehört der Umstand, dass unter den Bedingungen der repräsentativen Demokratie die politische Unterstützung für Reformen sehr oft von just jenen Akteuren gefordert ist, die unter den bestehenden Bedingungen vergleichsweise gut gefahren sind. Im Falle von Wahlsystemreformen betrifft dies besonders die regierenden Mehrheitsparteien, mit Blick auf expansive direktdemokratische Reformagenden praktisch sämtliche Akteure aus dem Lager der politischen Klasse, deren Handlungsautonomie und Gestaltungsmacht durch die Einführung von Volksrechten nachhaltig beschränkt würde. $\mathrm{Zu}$ den guten Nachrichten für die Demokratie gehören solche Befunde der empirischen Forschung, die zeigen, dass genuin demokratiefreundliche, gemeinschaftsorientierte Lösungen auch in üblicherweise machtpolitisch verminten Kontexten nicht ausgeschlossen sind. ${ }^{63}$

Unverzichtbar für erfolgreiche Systemreformen ist die Fähigkeit der verantwortlichen Akteure und ihrer Berater, ein hinreichendes Augenmaß und Gespür für die Kompatibilitäten und Inkompatibilitäten sektoraler Institutionenreformen bzw. Reformagenden zu entwickeln. Als besonders problembehaftet gilt vielen

61 Hinzukommen können freilich zahlreiche weitere Defekte der strukturellen Organisation des politischen Willensbildungs- und Entscheidungsprozesses, angesichts derer eine konzeptuelle und empirische Fokussierung allein auf den elektoralen Aspekt der liberalen Demokratie in der jüngeren Literatur zu Recht als unzureichend abgelehnt wird; Diamond, L.: The Democratic Rollback, in: Foreign Affairs, 87/2 (2008), 36-48.

62 LeDuc, L.: The Politics of Direct Democracy: Referendums in Global Perspective, Peterborough u.a., 2003; Freitag, M./Wagschal, U. (Hg.): Direkte Demokratie. Bestandsaufnahmen und Wirkungen im internationalen Vergleich, Berlin, 2007; Pallinger, Z.T. u. a. (Hg.): Direct Democracy in Europe. Developments and Prospects, Wiesbaden, 2007.

63 Gebauer, B.: Verfassungsreformprozesse in Großbritannien und der Schweiz. (Modell-)Demokratien im Wandel, Berlin/Wien, 2004; Katz, R.S.: Why Are There So Many (or So Few) Electoral Reforms?, in: Gallagher, M./Mitchell, P. (Hg.), The Politics of Electoral Systems, Oxford, 2005, 57-76. 
zu Recht der Import einzelner Institutionen aus grundlegend unterschiedlichen politisch-institutionellen Kontexten wie etwa die „Einpflanzung“ direktdemokratischer Instrumente schweizerischen Stils in Systemkontexte mit parlamentarischer Regierungsform. ${ }^{64}$ Aber selbst eine ähnliche institutionelle Basis bietet keinerlei Gewähr für eine fruchtbaren Transfer einzelner Institutionen wie die gegensätzlichen Erfahrungen mit dem System der Direktdemokratie und der Direktorialregierung in der Schweiz und in Uruguay belegen. ${ }^{65}$

Für alle größer angelegten Reformagenden gilt, dass Zielkonflikte zwischen unterschiedlichen demokratiepolitischen Agenden (wie zwischen der Erhöhung von Repräsentation und Inklusion einerseits und der Steigerung von Transparenz und Effizienz andererseits) letztlich unvermeidbalich sind. Ihre politische Lösung setzt eine bewusste Grundsatzentscheidung des Gemeinwesens zugunsten eines bestimmten Demokratiemodells voraus, mit Blick auf das Sinn und Nutzen von Reformoptionen kontextbezogen bestimmt werden können.

Die Hoffnung, durch ein aufwendiges Verfahren „beste Lösungen“ für die liberale Demokratie als solche zu finden, muss eine Illusion in den Köpfen von Sozialwissenschaftlern bleiben. $\mathrm{Zu}$ unterschiedlich sind die gesellschaftlichen Demokratievorstellungen politischer Gemeinwesen, selbst innerhalb einer auf den ersten Blick relativ autonomen Gruppe von Ländern wie den liberalen Demokratien Westeuropas. In Fragen der Gleichberechtigung zwischen Mann und Frau mag ein länderübergreifender Konsens gerade noch erreichbar sein, aber eine Entscheidung etwa über das „optimale“ Mischungsverhältnis zwischen der Handlungsautonomie der Regierung und der Kontrollmacht der Regierten über die Regierenden wird nur für den Einzelfall befriedigende Lösungen zulassen.

Selbst wenn diese Herausforderungen gemeistert sind und ein hinreichend breiter Basiskonsens über die grundlegenden Reformziele zwischen den maßgeblichen Entscheidungsträgern besteht, bleibt das Problem der politischen Umsetzung demokratiepolitischer Reformagenden. Die Abwesenheit eines Erkenntnisproblems ist in der Politik keineswegs gleichbedeutend mit der tatsächlichen Wahrnehmung einer Entscheidungs- und Gestaltungschance. ${ }^{66}$ Fallstudien unterstrei-

64 Schmidt, M. G.: Lehren der Schweizer Referendumsdemokratie, in: Offe, C. (Hg.), a. a. O., 111-123.

65 Altman, D.: Collegiate Executives and Direct Democracy in Switzerland and Uruguay: Similar Institutions, Opposite Political Goals, Distinct Results, in: Swiss Political Science Review, 14/3 (2008), 483520.

66 Sturm, R.: Föderalismusreform: Kein Erkenntnisproblem, warum aber ein Gestaltungs- und Entscheidungsproblem?, in: Politische Vierteljahresschrift, 46/2 (2005), 195-203. 
chen die große Bedeutung eines ernsthaften gesellschaftlichen Interesses an einer Reform, in dem ,politische Unternehmer“ wahlpolitisches Potenzial zu erkennen vermögen, welches anschließend in reformpolitische Maßnahmen überführt werden kann. ${ }^{67}$ Das mag auf den ersten Blick ernüchtern. Gleichwohl muss man weder Zyniker noch Pessimist sein, um zu den Merkmalen der liberalen Demokratie den Umstand zu zählen, dass die Bürger am Ende in beträchtlichem Maße jene Demokratie bekommen, die sie verdienen. 